\title{
Effective area calibration of the nuclear spectroscopic telescope array (NuSTAR)
}

Kristin K. Madsen, Walter Cook, Karl Forster, Brian Grefenstette, Fiona Harrison, et al.

Kristin K. Madsen, Walter Cook, Karl Forster, Brian Grefenstette, Fiona Harrison, Hiromasa Miyasaka, Sean Pike, "Effective area calibration of the nuclear spectroscopic telescope array (NuSTAR)," Proc. SPIE 10699, Space Telescopes and Instrumentation 2018: Ultraviolet to Gamma Ray, 106991W (6 July 2018); doi: 10.1117/12.2313675

EDIE Event: SPIE Astronomical Telescopes + Instrumentation, 2018, Austin, Texas, United States 


\title{
Effective Area calibration of the Nuclear Spectroscopic Telescope ARray (NuSTAR)
}

\author{
Kristin K. Madsen ${ }^{\mathrm{a}}$, Walter Cook $^{\mathrm{a}}$, Karl Forster ${ }^{\mathrm{a}}$, Brian Grefenstette ${ }^{\mathrm{a}}$, Fiona A. Harrison ${ }^{\mathrm{a}}$, \\ Hiromasa Miyasaka ${ }^{\mathrm{a}}$, and Seah Pike ${ }^{\mathrm{a}}$ \\ ${ }^{a}$ California Institute of Technology, 1200 E. California Blvd, Pasadena, USA
}

\begin{abstract}
The Nuclear Spectroscopic Telescope ARray (NuSTAR) has been in orbit for 6 years, and with the calibration data accumulated over that period we have taken a new look at the effective area calibration. The NuSTAR $10-\mathrm{m}$ focal length is achieved using an extendible mast, which flexes due to solar illumination. This results in individual observations sampling a range of off-axis angles rather than a particular off-axis angle. In our new approach, we have split over 50 individual Crab observations into segments at particular off-axis angles. We combine segments from different observations at the same off-axis angle to generate a new set of synthetic spectra, which we use to calibrate the vignetting function of the optics against the canonical Crab spectrum.
\end{abstract}

Keywords: NuSTAR, X-ray, Satellite

\section{INTRODUCTION}

The Nuclear Spectroscopic Telescope Array (NuSTAR) was launched in June $2012^{1}$ and carries two co-aligned conical grazing incidence Wolter-I approximation ${ }^{2}$ optics modules (OMA, and OMB) that focus onto two identical focal plane modules (FPMA and FPMB), each composed of four solid state CdZnTe pixel detector arrays (enumerated DET0 through DET3) with a minimum detector threshold of $3 \mathrm{keV} .{ }^{3,4}$ There are 133 shells in each optic, and the outer 43 shells are coated with a W/Si multilayer while the inner 90 shells are coated with $\mathrm{Pt} / \mathrm{C}$, limiting the highest efficient reflective X-ray energies to the Pt $78.4 \mathrm{keV}$ K-edge. ${ }^{5}$

The instrument has been in orbit for 6 years, and the original effective area calibration, reported in Madsen et al (2015), ${ }^{6}$ was performed during the first year of operation. In 2016 there was an update, as a result of a new gain correction and the re-calibration of the detector absorption parameters, which were reported in Madsen et al. (2017). ${ }^{7}$ Both these calibrations were generated the same way, which we name the off-axis angle weighting method, or 'oaa-weighting' for short, and summarize later. This calibration has worked well, but over the years, certain features inherent to this particular method have emerged. Two examples are shown in Figure 1: on the left there is occasionally a low energy tail that becomes evident when comparing to other instruments, and on the right, with bright sources in particular, features sometimes show themselves at $\sim 11-12 \mathrm{keV}$ and at around $\sim 26-29 \mathrm{keV}$, as well as some deviations between the two modules at low energy. Overall, these features are on the few percent level.

In this paper we present a new approach to address these residual features and improve the overall calibration.

\section{CALIBRATING THE EFFECTIVE AREA}

Preferably, instruments should be understood by their ground calibration and only require some additional inorbit calibration to verify the ground results. Reality, however, has shown that instruments rarely perform exactly as they did on the ground, sometimes related to the finite source sizes and lengths of calibration facilities compared to infinitely distant point sources on the sky, or due to a process that happened after the ground calibration, which may or may not be continual. In some cases, corrections can be made based on an understood physical process, such as a time dependent contamination layer, missing absorption component, or a geometrical

Further author information: (Send correspondence to K.K.M.)

K.K.M.: E-mail: kristin@srl.caltech.edu, Telephone: 16263956634

Space Telescopes and Instrumentation 2018: Ultraviolet to Gamma Ray, edited by Jan-Willem A. den Herder, Shouleh Nikzad, Kazuhiro Nakazawa, Proc. of SPIE Vol. 10699, 106991W · @ 2018 SPIE CCC code: $0277-786 X / 18 / \$ 18 \cdot$ doi: $10.1117 / 12.2313675$ 

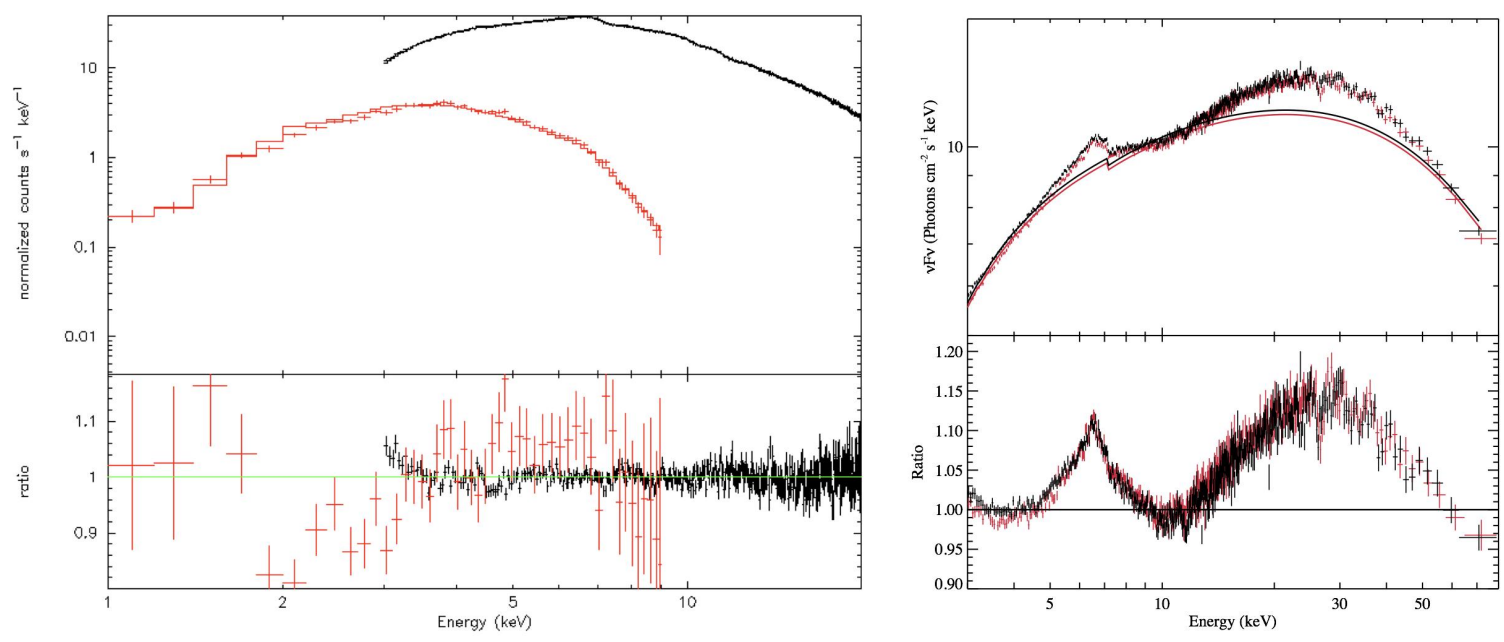

Figure 1. Left: Simultaneous fit to NuSTAR and Swift/XRT sometimes reveal low energy tails. Right: In bright sources absorption line features occasionally appear at around $\sim 11-12 \mathrm{keV}$ and $\sim 26-29 \mathrm{keV}$, as well as some deviations between the two modules at low energy.

correction from the calibration facility to space application. But in other cases, the underlying physical process, or geometry, may not be known, or be too complex to accurately model.

For NuSTAR, the issue resides in the complexities of the multi-layer response. There are 10 different multilayer recipes, ${ }^{5}$ approximately evenly distributed across $\sim 3000$ individual segments of glass, and although the theoretical responses of the recipes are known, and we know where each segment resides, during production depth variations occured in the recipes, and since we did not measure the responses of individual pieces, which is highly dependent on grazing incidence angle, we do not know the actual response of each piece. Deviations from the theoretical response of the full optic were therefore expected and directly related to the differences in the multi-layer recipes. However, because the parameter space is so large, 3000 individual mirrors times their response as a function of off-axis angle, it was not practical to solve for the individual multi-layer responses.

It was therefore necessary to calibrate the effective area of the NuSTAR optics against a standard candle. We used the Crab, which is a center filled pulsar wind nebula (PWN) powered by a pulsar with a double peaked profile of period $\mathrm{P} \sim 33 \mathrm{~ms}$. It has served as the primary celestial calibration source for many hard X-ray instruments because of its brightness, relative stability, and simple power-law spectrum over the band from $1-100 \mathrm{keV}{ }^{8}$ The spatially phase-averaged integrated spectrum of the Crab nebula+pulsar in the 1-100 keV X-ray band has been well-described by a power-law with photon index of $\Gamma \sim 2.1,{ }^{8}$ while above $100 \mathrm{keV}$ the hard X-ray instruments (INTEGRAL/SPI/ISGRI, CGRO) measure a softer index of $\Gamma \sim 2.20-2.25$. The exact location of the turnover is not well determined, since it appears very gradual and there is no analytical model to describe it, but it is estimated to occur somewhere between $50-120 \mathrm{keV} .{ }^{9}$ Weisskopf et al $(2010)^{10}$ did a study with $R X T E / \mathrm{PCA}$, $X M M$, and $A S C A$, looking for deviations from a power-law in the $0.2-50 \mathrm{keV}$ energy range, and concluded that within the precision of the available instrumentation there is no detectable bend in the phase-averaged integrated spectrum of the Crab. Measurements using NuSTAR stray-light data support these findings. ${ }^{7}$ The stability of the Crab has been tracked in great detail over the last decade and during this period the flux has been observed to change by $7 \%$ across the $10-100 \mathrm{keV}$ bandpass. ${ }^{11}$ The change, however, is slow, and the deviation in flux per year over the period it has been observed is only $\sim 3 \%$. The spectral index has also been observed to vary peak-to-peak by $\Delta \Gamma \sim 0.025$ in $R X T E / \mathrm{PCA},{ }^{12}$ but as with the flux variation it is slow, and on average over the 16 years the Crab has been observed, it has remained steadily at $\Gamma \sim 2.1$.

Ideally, a calibration source should remain completely stable, but the X-ray sky is not known for its quietscent behavior, and since the systematic errors of the instrument from measurement to measurement are on the order of $\sim 5 \%,{ }^{6}$ the intrinsic variability of the Crab is acceptable for our purpose. 
The task of the effective area calibration is thus to compare the measured spectrum to the assumed Crab model and infer a correction function, $\operatorname{Corr}(E, \theta)$, to the effective area response, and in the following sections we will first present the methodology used to obtain this function, and then describe the details of the data analysis.

\subsection{Methodology}

Mathematically, we model the detected counts in a given instrumental pulse height bin, $C(P I, \theta)$, according to the equation,

$$
C(P I, \theta)=\int \frac{d N(E)}{d E} R(P I, E, \theta) d E .
$$

Here $d N(E) / d E$ is the model differential photon spectrum of the observed target as a function of incident photon energy, $E$, and $R(P I, E, \theta)$ is the response matrix that captures the photon incident in a given pulse height bin, $P I$, at an off-axis angle, $\theta$. In practice, this integral is approximated as a finite sum by sampling $R(P I, E, \theta)$ on a fine grid. The off-axis angle, $\theta$, is the angle of incoming X-rays with respect to the optical axis of the mirrors. As the optical axis moves with respect to the detector position during an observation, the modeled response is sampled on a finite time grid and then summed for a given exposure.

As is typical for X-ray astronomy missions, the response matrix is divided into two components,

$$
R(P I, E, \theta)=\operatorname{RMF}(P I, E) A(E, \theta) .
$$

$\operatorname{RMF}(P I, E)$ is known as the redistribution matrix, which contains detector quantum efficiency and resolution effects, and is unitless (a fraction between 0 and 1, where a value of 1 indicates $100 \%$ quantum efficiency). The quantity $A(E, \theta)$ is the effective area, also known as the ancillary response function (ARF), which captures the effective area of the mirror optics, as well as several other factors unique to NuSTAR,

$$
A(E, \theta)=A_{o}(E) V(E, \theta) \operatorname{detabs}(E) \operatorname{GR}(E, \theta) \operatorname{AS}(E, \theta) \operatorname{Corr}(E, \theta) .
$$

Here $A_{0}(E)$ is the modeled effective area of the mirror segments estimated from theoretical ray-tracing simulations, and $V(E, \theta)$ is the geometric vignetting function, also based on ray-tracing simulations. The quantities detabs, GR, and AS are the detector dead layer absorption, ghost ray correction, and aperture stop correction, respectively (for details on these components see Madsen et al $(2015)^{6}$ and Madsen et al $(2017)^{13}$ ). $\operatorname{Corr}(E, \theta)$ is the empirically derived correction factor we want to obtain.

In practice, discovering the correction function $\operatorname{Corr}(E, \theta)$, involves taking numerous observations of the Crab at different, fixed off-axis angles. In this way a grid of countrates at $E$ and $\theta$ are obtained and can be used to interpolate the response at any desired point, where, obviously, the finer the grid the more accurate the function.

With NUSTAR, however, obtaining such a data set is problematic. The observatory has two benches separated by 10.14 meters, which move relative to each other due to motions of the mast that connects them and causes the optical axis to travel across the FPMs by up to several arcminutes. This means, that each observation covers a range of off-angles that are unique for that observation only, as shown by Figure 2 (left) for observation 10013033004. In this particular observation, the off-axis angle distribution covers more than 2 arcminutes and, except for a narrow range, there is not sufficient signal to measure the spectrum accurately as a function of off-axis angle. To overcome this problem, we add together many such observations to increase statistics, and in our original oaa-weighted method we did that by calculating the mean off-axis angle for each observation and assigning the observations to one arcminute bins: $0-1^{\prime}, 1-2^{\prime}, \ldots, 6-7^{\prime}$. For each bin the assigned observations were then combined, and a new off-axis angle for the combined effective area was calculated by weighting the individual off-axis angle distributions.

The issue with this method, is that the combined distributions were actually overlapping as shown in Figure 2 (right), and that the spectra are sampling a range of off-axis angles that go beyond the assigned bin. Also, because the bins are required to be so large to get enough statistics, our knowledge of what happens in between is poor. Figure 3 (left) shows the effective area correction factor, $C(E, \theta)$ for energies $3.0,10.1$, and $46.3 \mathrm{keV}$, and although to first approximation the correlation appears linear as a function of off-axis angle, there is substructure at 3.0 and $10.1 \mathrm{keV}$ between $2-5^{\prime}$. 

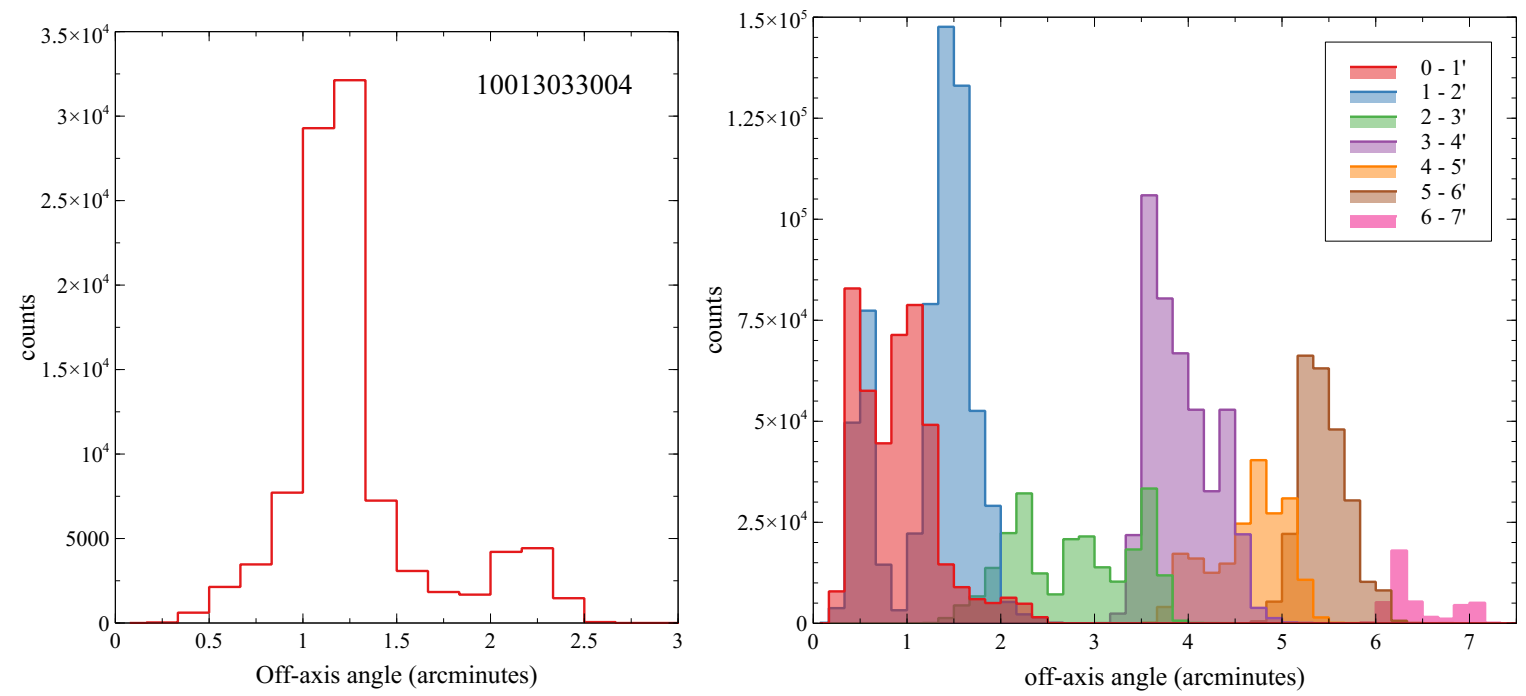

Figure 2. Left: Histogram of the count distribution between $3-78 \mathrm{keV}$ as a function of off-axis angle for a single observation. Right: Histogram of the 'oaa-weighting' of the original binning using the $\sim 35$ observations available at the time. ${ }^{6}$
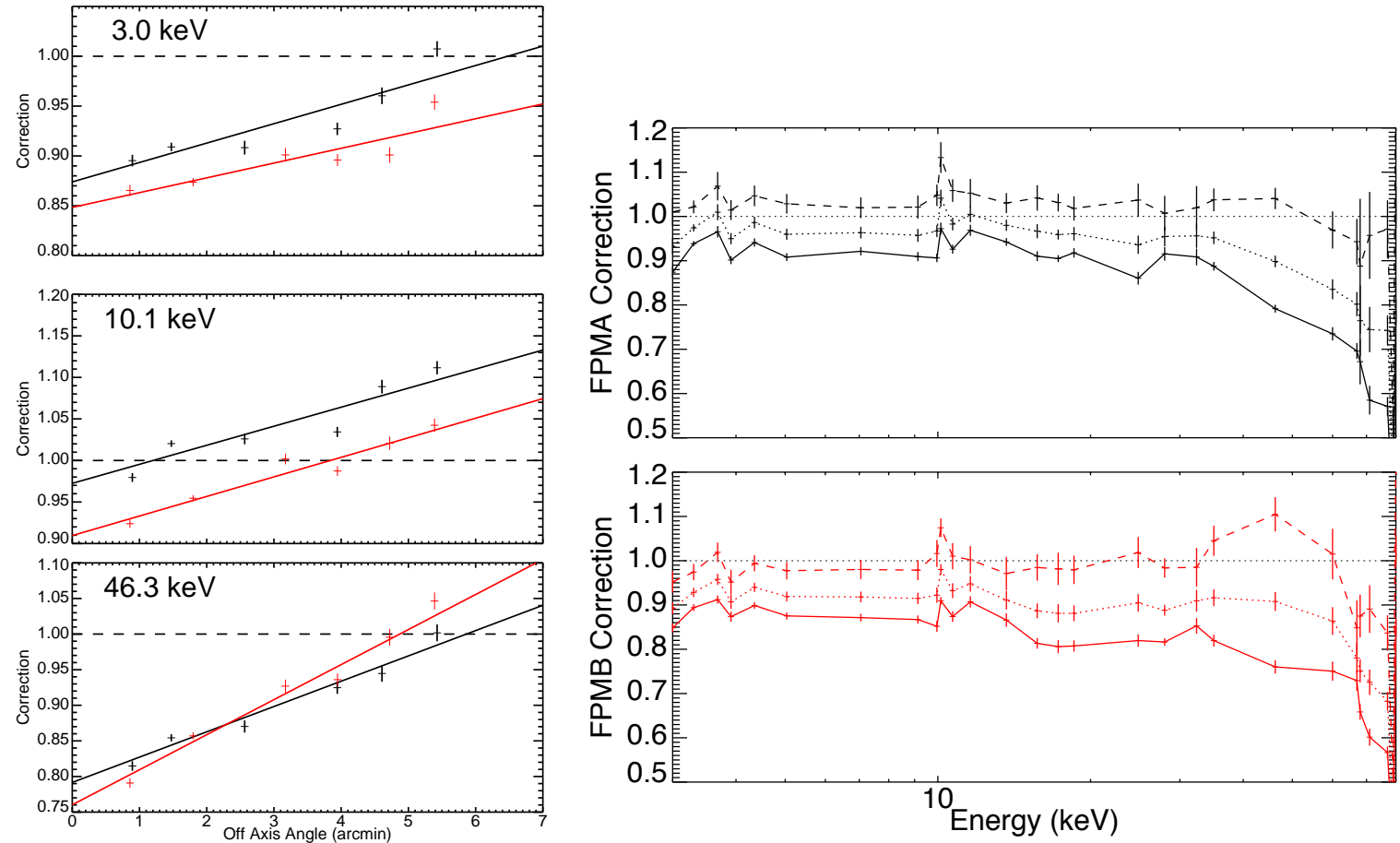

Figure 3. Correction functions, $C(\theta, E)$, of the current $N u S T A R$ calibration. Left: The linear (in $\theta)$ function for selected energies. Right: $C(\theta, E)$ for offaxis angles $0^{\prime}$ (solid), $3^{\prime}$ (dotted), and $7^{\prime}$ (dashed). 

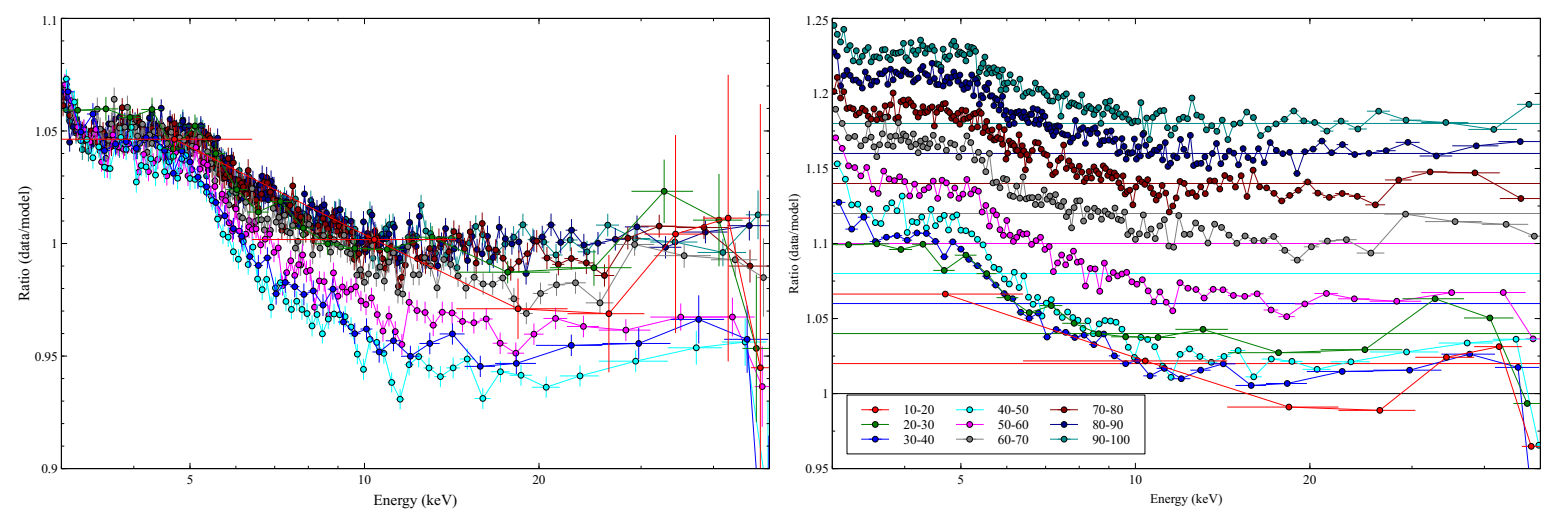

Figure 4. Ratio of the data to the Crab model for off-axis bins 10-100". Left: The curves maintained at their absolute value. Right: the curves have been offset for clarity. Systematic variations from bin to bin is evident.

\subsubsection{New Method}

To address these issues, we have devised a new method. The number of Crab observations has grown from 39 to 53, with almost a factor of 2 increase in exposure time, and this allows for a higher fidelity analysis than was previously available. The most important change is that we calculate the off-axis angle of each individual photon. Due to the extent of the Crab and the PSF, we do not measure the actual photon's position, but the off-axis angle of the source center at the time of the photon's arrival. In this manner, we can assign each photon to a specific off-axis angle bin without having overlapping distributions.

Since the angular resolution of the response files is $10^{\prime \prime}$, we subdivide each observation into $10^{\prime \prime}$ bins. We then combine all observations from the same off-axis angle bin together in a synthetic spectrum, which we can then fit against the reference Crab spectrum to obtain the correction for each $10^{\prime \prime}$ bin instead of each arcminute. Figure 4 shows the ratio spectra of off-axis angle bins 10-100", and systematic non-linear changes across the sub-arcminute bin can be clearly seen. We note here, that the observed deviations from the Crab spectrum, particularly at low energies, shown here are more severe than the actual current calibration displays. The reason for this is that included here is an adjustment to the detector absorption parameters, which affects the low energies.

In addition to the major change above, we have also improved the backgrounds, the detector absorption coefficients, and the fitting procedure, the details of which will be discussed in the analysis section.

\section{ANALYSIS}

\subsection{Observations and Data Reductions}

The on-going NUSTAR Crab calibration campaign measures the Crab spectrum at various off-axis angles. This has resulted in the 53 individual observations listed in Table 1 and a total exposure time of 174 ks. Figure 5 shows the distribution of all counts within the $3-78 \mathrm{keV}$ band as a function of off-axis angle for FPMA and FPMB. Most of the observations are at the typical 1.5' for 'on-axis' position (moving closer to the optical axis causes the source to fall into detector gaps), but overall we have $2-3 \times 10^{6}$ counts per off-axis bin. The reason for FPMB having an overall lower total number of counts is that it has a lower effective area.

Despite the angular extent of the Crab of $\sim 120^{\prime \prime} \times 100^{\prime \prime}$, we treat it as a point source since the center of the nebula where the pulsar resides dominates the emission. The difference in the effective area response between using a point source effective area centered on the pulsar and a count-rate adjusted extended effective area is only $\sim 1-2 \%$ and therefore an acceptable error. Most importantly, treating the Crab as a point source allows us to use the built-in pipeline corrections for the aperture stop, ghost ray and PSF corrections (for details on these components see Madsen et al. $(2015)^{6}$ and $\left.(2017)^{7,13}\right)$. These corrections are not applicable to extended source responses, and since they can be larger than the error introduced by assuming the Crab is a point source, they are extremely important to include. We extract counts from a $200^{\prime \prime}$ region, which includes $95 \%$ of all photons in 


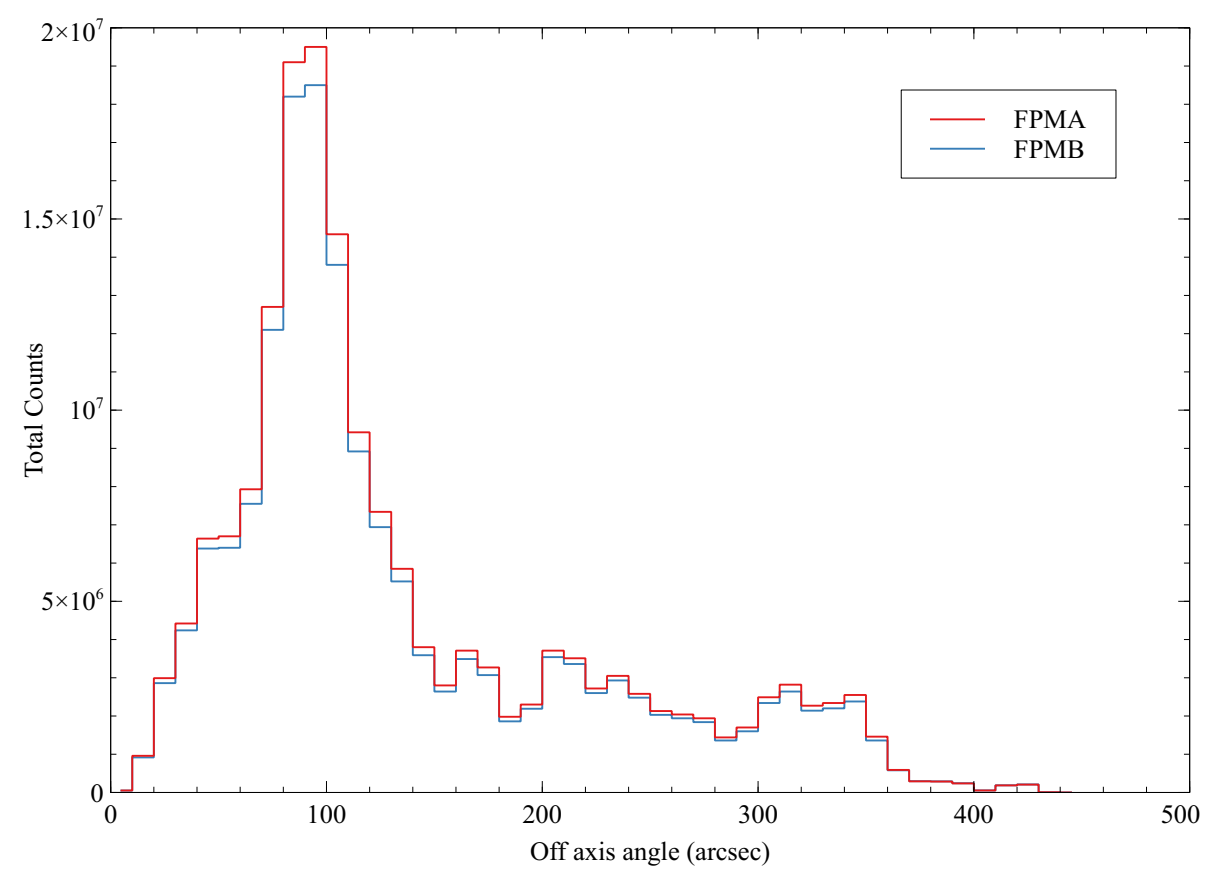

Figure 5. Histogram of the count distribution between $3-78 \mathrm{keV}$ as a function of off-axis angle.

the source extraction region, and use the NuSTARDAS pipeline version v1.8.0 to reduce the data, generate the detector response, and apply all corrections to the base effective area.

We combine the spectra and their backgrounds into the final off-axis angle bin using the FTOOL addascaspec.

\subsubsection{Off-axis angle filtering}

The most important aspect of the data preparation is the assignment of the counts into the appropriate off-axis bins. We use an off-axis file that contains the time dependent history of the off-axis angle, generated with the task nuproducts for the entire observation, to make angle specific good time interval (GTI) files. These GTI files we provide as input into nuproducts to split the observations into the angle specific spectra.

\subsubsection{Background}

Obtaining backgrounds for the Crab observations presented us with some issues. Background starts to affects the spectrum at $\sim 55 \mathrm{keV}$, which at these energies is mostly internal detector background. However, because of the Crab's brightness and its PSF extent, we cannot extract a local background from the same observation. Along with the stray-light observations mentioned above, we take blank backgrounds a few degrees from the Crab. Utilizing these blank fields, we extracted backgrounds from the same location on the detector as for the observation.

Because the projection of the detector on the sky is never precisely the same for two observations, we have to remove the spacecraft jitter from the background observations. This is a non-standard procedure, which involves correcting files that track the center of the detector on the sky to produce a jitter-free image. In this manner, we combine together several jitter-corrected background observations listed in Table 2, into a master background. By definition when filtering on off-axis angle, the source position on the detector is stable and virtually jitter-free (since the spacecraft/mast jitter is what causes the off-axis angle variations), and we can therefore extract the background from the same location on the detector where the source falls.

For each extracted spectrum, we get a background that has an exposure time of $60 \mathrm{ks}$. 
Table 1. Observing Log

\begin{tabular}{|c|c|c|c|c|c|}
\hline Obsid & $\begin{array}{c}\text { mean off-axis angle } \\
\text { (arcmin) }\end{array}$ & $\begin{array}{c}\text { exposure time } \\
(\mathrm{ks})\end{array}$ & Obsid & $\begin{array}{c}\text { mean off-axis angle } \\
\text { (arcmin) }\end{array}$ & $\begin{array}{c}\text { exposure time } \\
(\mathrm{s})\end{array}$ \\
\hline 10013021002 & 3.6 & 2275 & "10013022002 & 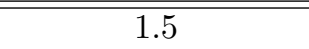 & 2592 \\
\hline 10013022004 & 1.5 & 2347 & 10013022006 & 1.4 & 2587 \\
\hline 10013023002 & 4.1 & 2102 & 10013024002 & 4.7 & 2258 \\
\hline 10013025002 & 5.0 & 1235 & 10013025004 & 5.0 & 1161 \\
\hline 10013025006 & 4.8 & 1593 & 10013026002 & 5.5 & 2540 \\
\hline 10013026004 & 5.7 & 1162 & 10013027002 & 5.8 & 1182 \\
\hline 10013027004 & 5.6 & 1105 & 10013028002 & 6.5 & 1601 \\
\hline 10013028004 & 6.7 & 1254 & 10013029001 & 4.4 & 2909 \\
\hline 10013030001 & 4.4 & 3023 & 10013031002 & 1.9 & 2507 \\
\hline 10013032002 & 1.3 & 2595 & 10013033002 & 1.5 & 1383 \\
\hline 10013033004 & 1.3 & 1269 & 10013034002 & 1.2 & 988 \\
\hline 10013034004 & 0.8 & 5720 & 10013034005 & 0.9 & 5968 \\
\hline 10013035002 & 5.4 & 9401 & 10013036002 & 5.8 & 179 \\
\hline 10013037002 & 2.0 & 2679 & 10013037004 & 2.9 & 2796 \\
\hline 10013037006 & 2.9 & 2799 & 10013037008 & 3.1 & 2814 \\
\hline 10013038002 & 3.9 & 3084 & 10013038004 & 3.9 & 2217 \\
\hline 10013038006 & 4.0 & 266 & 10013038008 & 4.0 & 2231 \\
\hline 10013039002 & 4.1 & 590 & 10013039003 & 4.2 & 583 \\
\hline 80001022002 & 1.5 & 3917 & 10002001002 & 1.6 & 2608 \\
\hline 10002001004 & 1.6 & 2386 & 10002001006 & 1.4 & 14263 \\
\hline 10002001008 & 1.6 & 4941 & 10002001009 & 1.3 & 5198 \\
\hline 10202001002 & 1.1 & 1454 & 10202001004 & 2.0 & 9025 \\
\hline 10202001006 & 1.7 & 4307 & 10202001007 & 2.9 & 4104 \\
\hline 10302001002 & 2.5 & 4300 & 10302001004 & 1.2 & 7547 \\
\hline 10302001006 & 1.0 & 3729 & 10402001002 & 1.1 & 9436 \\
\hline 10402001004 & 1.4 & 3312 & 10402001006 & 1.4 & 4757 \\
\hline 10402001008 & 1.3 & 5657 & & & \\
\hline
\end{tabular}

Table 2. Background

\begin{tabular}{cc}
\hline Obsid & $\begin{array}{c}\text { exposure time } \\
(\mathrm{s})\end{array}$ \\
\hline \hline 10311002002 & 3091 \\
10311002004 & 19627 \\
10311002006 & 14898 \\
10311002008 & 18633 \\
10210002002 & 20567 \\
10210002003 & 21143 \\
10402006002 & 17568 \\
\hline
\end{tabular}


Table 3. detabs parameters

\begin{tabular}{c|c|c|c}
\hline Module & Detector & Pt $(\mu \mathrm{m})$ & CZT $(\mu \mathrm{m})$ \\
\hline \hline A & 0 & 0.095 & 0.225 \\
A & 1 & 0.091 & 0.295 \\
A & 2 & 0.074 & 0.370 \\
A & 3 & 0.095 & 0.276 \\
\hline B & 0 & 0.106 & 0.214 \\
B & 1 & 0.065 & 0.295 \\
B & 2 & 0.082 & 0.246 \\
B & 3 & 0.071 & 0.261 \\
\hline
\end{tabular}

\subsubsection{Detector Absorption Correction}

A Pt contact coating and a CdZnTe dead layer on the detector surface cause absorption at low energies. No ground calibration of the absolute efficiency at these energies was performed, and the thickness of the layers needs to be measured in orbit. We built an XSPEC absorption model with cross-sections created by Geant4, and the adopted photon interaction model is the Livermore low-energy EM model based on the evaluated photon data library, EPDL97. ${ }^{14}$ We use Crab stray-light observations, which are observations where the source flux bypasses the optics and falls directly on the detectors, since with these observations we can remove the uncertainties of the optics and obtain a particularly simple response that allows us to measure the Crab flux to $4 \%{ }^{7}$ NuSTAR has a legacy program monitoring the Crab in this configuration, and we use this data set to measure the detector absorption thickness of the $\mathrm{Pt}$ and CdZnTe. As more and more data is collected, the precision is increased, and for the process used in this paper we used the numbers summarized in Table 3.

\subsection{Fitting procedure}

As discussed above, the correction function we desire to obtain is a multiplicative factor that gets applied to the effective area. In practical terms, we model the differential photon spectral model of the phase-averaged integrated spectrum of pulsar+nebula as a simple absorbed power law,

$$
\frac{d N(E)}{d E}=\operatorname{tbabs}(E) N E^{-\Gamma} \mathrm{ph} \mathrm{s}^{-1} \mathrm{~cm}^{-2} \mathrm{keV}^{-1}
$$

Here $E$ is the photon energy, tbabs is the interstellar absorption using Wilms abundances ${ }^{15}$ and Verner crosssections, ${ }^{16} \Gamma$ is the power-law photon index, and $N$ is the normalization factor. When $\Gamma=2.1$ and $\mathrm{N}=8.7 \mathrm{keV}^{-1}$ $\mathrm{cm}^{-2} \mathrm{~s}^{-1}$ at $1 \mathrm{keV}$, we refer to this as the canonical Crab model. We fold this model through the known response functions to obtain a model count spectrum for a fixed off-axis angle, $\theta$,

$$
C(P I)_{\text {model }}=\operatorname{RMF}(P I, E) \operatorname{ARF}(E) C o r r(E),
$$

where $\operatorname{ARF}(E)$ contains all the effective area corrections discussed in Section 3.1, and $\operatorname{Corr}(E)$ is the correction function we wish to find. We compare this model count spectrum to the actual spectrum and use least squares fitting of a piece-wise linear function to find the array elements of $\operatorname{Corr}(E)$. We do this for all $\theta$ to obtain $\operatorname{Corr}(\theta, E)$

This forward folding approach is necessary because the RMF is a non-diagonal matrix, which cannot be inverted. This also cautions that the solution to $\operatorname{Corr}(E, \theta)$ is not unique, and care must be taken to avoid nonphysical solutions. These may occur from high energy down-scattered photons when the fit, due to large errors on the countrate, may take on unrealistically large values. This issue may be mitigated by choosing the right binning and ensuring sufficient statistics to an appropriate grid of fitting points. Previously, we used an energy correction grid (see Figure 3, left) that we identified based on the residual features we observed. It ensured a fairly smooth broad band correction, but did poorly for narrow features. To target these features, which emerge between 10-40 keV, and allow for a finer correction at low energies, we employ in this version a logarithmic binning in energy that varies from 0.03-0.1, depending on the fidelity of the fitted spectrum, across three sub bands: $3-20,20-40,40-78 \mathrm{keV}$. We find that grouping the counts by $200-400$ yields good results. 
As shown by Figure 4, the energy bins grow large above $\sim 40 \mathrm{keV}$, and not shown are the $50-78 \mathrm{keV}$ spectra, which have very few bins. It is therefore, unfortunately, not feasible to fit above $\sim 40 \mathrm{keV}$ with $10^{\prime \prime}$ bins even with the additional exposure time. To get enough statistic at these energies, we have to return to the original $1^{\prime}$ off-axis bins and have devised a two stage approach:

- combine $10^{\prime \prime}$ spectra into $1^{\prime}$ bins of $0-1^{\prime}, 1-2^{\prime}, \ldots, 6-7^{\prime}$

- fit the $1^{\prime}$ spectra from $3-78 \mathrm{keV}$

- correct the vignetting function and re-extract products

- fit the new $10^{\prime \prime}$ spectra between $3-30 \mathrm{keV}$

The first part is focused on correcting the overall broadband shape, and the second focused on the fine scale corrections below $30 \mathrm{keV}$.

Figure 6 shows the process illustrated with select $10^{\prime \prime}$ and $1^{\prime}$ spectra. After the first iteration, where we have fit the correction function to the $1^{\prime}$ spectra ('A'), it may be seen that the residuals are much improved ('B' and ' $\mathrm{E}$ '), but deviations are still observed, even in the $1^{\prime}$ spectra on which the correction was based ('B'). This is because the correction function is measured for a specific off-axis angle, when it really covers the entire $1^{\prime}$ bin. To correct for this effect we fit the $10^{\prime \prime}$ spectra (' $\mathrm{E}$ ') and obtain the final spectra shown in panel ' $\mathrm{C}$ ' and ' $\mathrm{F}$ '.

Figure 7 shows the comparison between the current calibration from CALDB version 20170727 (red curve, triangles) and the new calibration using the procedure described in this paper. The effect is on the $\sim 2 \%$ level, which may appear small, but is noticeable between $10-20 \mathrm{keV}$, where it has corrected the features that were previously there.

\section{CONCLUSION AND FUTURE PROSPECTS}

We have presented a new and improved method for calibrating the effective area of NuSTAR. We have validated the end-to-end procedure and verified that we get an improvement in the desired $20-40 \mathrm{keV}$ area. The procedure, however, is not yet ready for release. We first need to decide on the values of the 'canonical Crab'. In this paper we used $N=8.7$ and $\Gamma=2.1$, because that is what has been used for the current calibration, but with the $N u S T A R$ stray-light observations we find the values to be $N=9.7 \pm 0.2$ and $\Gamma=2.11 \pm 0.01 .{ }^{7}$ The expected Crab flux variations are on the order of $3 \%$ per year, and because of the spread in time over which the observations were taken, we obviously cannot pin the normalization to a specific date. Since 2017 we have been taking straylight observations together with focused observations, and we will most likely use these to derive an average normalization.

\section{REFERENCES}

[1] Harrison, F. A., Craig, W. W., Christensen, F. E., and et al., "The Nuclear Spectroscopic Telescope Array (NuSTAR) High-energy X-Ray Mission," ApJ 770, 103 (June 2013).

[2] Petre, R. and Serlemitos, P., "Conical Imaging Mirrors for High-Speed X-ray Telescopes," Applied Optics 24, 1833-1837 (1985).

[3] Rana, V. R., Cook, III, W. R., Harrison, F. A., Mao, P. H., and Miyasaka, H., "Development of focal plane detectors for the Nuclear Spectroscopic Telescope Array (NuSTAR) mission," in [Proc. SPIE], 7435, 3 (Aug. 2009).

[4] Kitaguchi, T., Grefenstette, B. W., Harrison, F. A., Miyasaka, H., Bhalerao, V. B., Cook, III, W. R., Mao, P. H., Rana, V. R., Boggs, S. E., and Zoglauer, A. C., "Spectral calibration and modeling of the NuSTAR CdZnTe pixel detectors," in [Proc. SPIE], 8145 (Sept. 2011).

[5] Madsen, K. K., Harrison, F. A., Mao, P. H., Christensen, F. E., Jensen, C. P., Brejnholt, N., Koglin, J., and Pivovaroff, M. J., "Optimizations of $\mathrm{Pt} / \mathrm{SiC}$ and W/Si multilayers for the Nuclear Spectroscopic Telescope Array," in [Proc. SPIE], $\mathbf{7 4 3 7}$ (Aug. 2009). 

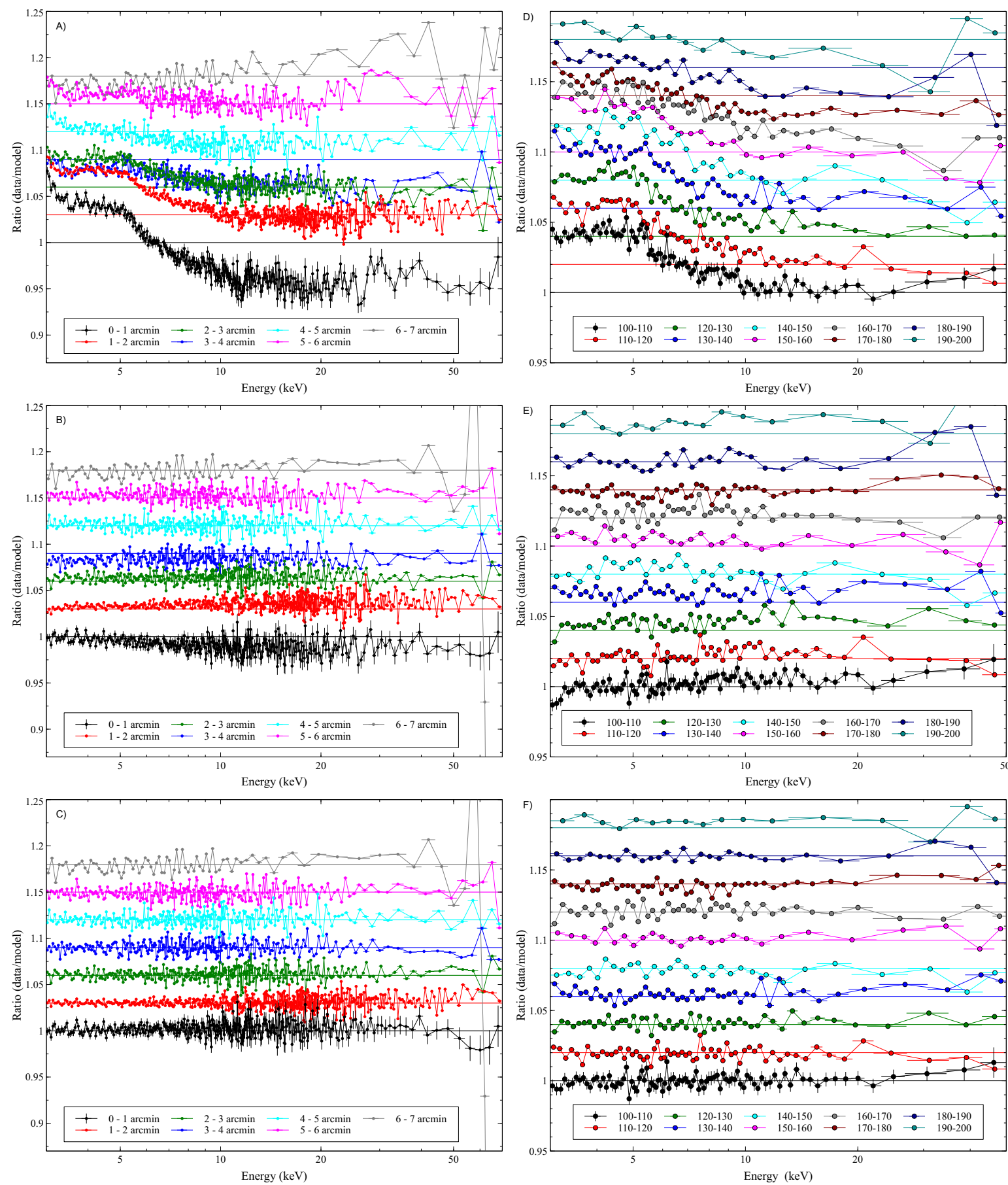

Figure 6. Synthetic $1^{\prime}$ (left column) and $10^{\prime \prime}$ (right column) spectra. A and D are the base responses before fitting. B and $\mathrm{E}$ are after fitting the $1^{\prime}$ in $\mathrm{A}$ spectra. $\mathrm{C}$ and $\mathrm{F}$ are after fitting the $10^{\prime}$ spectra in $\mathrm{E}$. 


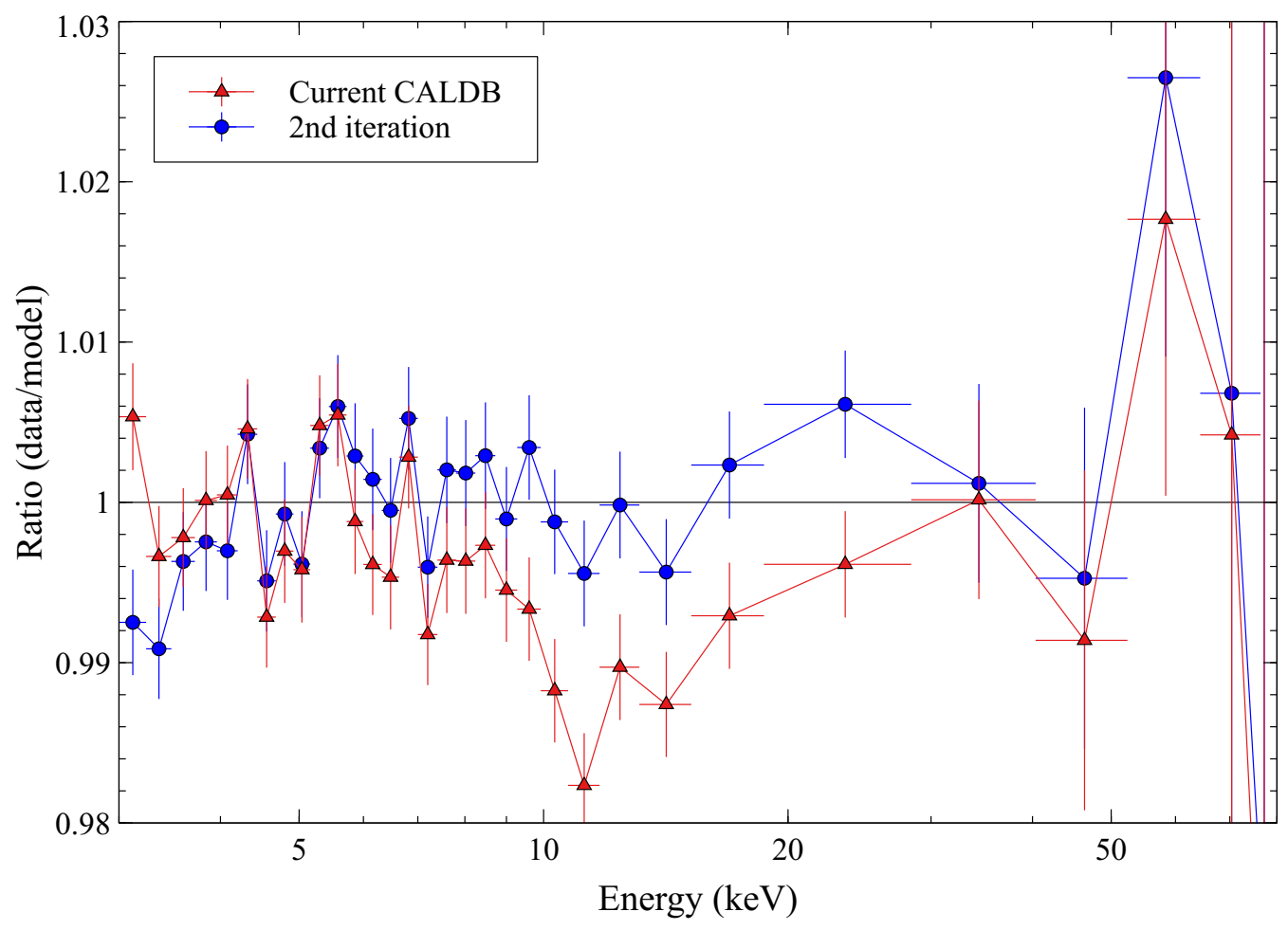

Figure 7. Comparison between current calibration and new effective area calibration.

[6] Madsen, K. K., Harrison, F. A., Markwardt, C. B., An, H., Grefenstette, B. W., Bachetti, M., Miyasaka, H., Kitaguchi, T., Bhalerao, V., Boggs, S., Christensen, F. E., Craig, W. W., Forster, K., Fuerst, F., Hailey, C. J., Perri, M., Puccetti, S., Rana, V., Stern, D., Walton, D. J., Jørgen Westergaard, N., and Zhang, W. W., "Calibration of the NuSTAR High-energy Focusing X-ray Telescope.," ApJS 220, 8 (Sept. 2015).

[7] Madsen, K. K., Forster, K., Grefenstette, B. W., Harrison, F. A., and Stern, D., "Measurement of the Absolute Crab Flux with NuSTAR," ApJ 841, 56 (May 2017).

[8] Kirsch, M. G., Briel, U. G., Burrows, D., Campana, S., Cusumano, G., Ebisawa, K., Freyberg, M. J., Guainazzi, M., Haberl, F., Jahoda, K., Kaastra, J., Kretschmar, P., Larsson, S., Lubiński, P., Mori, K., Plucinsky, P., Pollock, A. M., Rothschild, R., Sembay, S., Wilms, J., and Yamamoto, M., "Crab: the standard x-ray candle with all (modern) x-ray satellites," in [Proc. SPIE], Siegmund, O. H. W., ed., 5898, 22-33 (Aug. 2005).

[9] Jourdain, E. and Roques, J. P., "The High-Energy Emission of the Crab Nebula from $20 \mathrm{keV}$ TO $6 \mathrm{MeV}$ with Integral SPI," ApJ 704, 17-24 (Oct. 2009).

[10] Weisskopf, M. C., Guainazzi, M., Jahoda, K., Shaposhnikov, N., O’Dell, S. L., Zavlin, V. E., Wilson-Hodge, C., and Elsner, R. F., "On Calibrations Using the Crab Nebula and Models of the Nebular X-Ray Emission," ApJ 713, 912-919 (Apr. 2010).

[11] Wilson-Hodge, C. A., Cherry, M. L., Case, G. L., Baumgartner, W. H., Beklen, E., Narayana Bhat, P., Briggs, M. S., Camero-Arranz, A., Chaplin, V., Connaughton, V., Finger, M. H., Gehrels, N., Greiner, J., Jahoda, K., Jenke, P., Kippen, R. M., Kouveliotou, C., Krimm, H. A., Kuulkers, E., Lund, N., Meegan, C. A., Natalucci, L., Paciesas, W. S., Preece, R., Rodi, J. C., Shaposhnikov, N., Skinner, G. K., Swartz, D., von Kienlin, A., Diehl, R., and Zhang, X.-L., "When a Standard Candle Flickers," ApJL 727, L40 (Feb. 2011).

[12] Shaposhnikov, N., Jahoda, K., Markwardt, C., Swank, J., and Strohmayer, T., "Advances in the RXTE Proportional Counter Array Calibration: Nearing the Statistical Limit," ApJ 757, 159 (Oct. 2012). 
[13] Madsen, K. K., Christensen, F. E., Craig, W. W., Forster, K. W., Grefenstette, B. W., Harrison, F. A., Miyasaka, H., and Rana, V., "Observational artifacts of nuclear spectroscopic telescope array: ghost rays and stray light," Journal of Astronomical Telescopes, Instruments, and Systems 3, 3-3-13 (2017).

[14] Cirrone, G. A. P., Cuttone, G., Di Rosa, F., Pandola, L., Romano, F., and Zhang, Q., "Validation of the Geant4 electromagnetic photon cross-sections for elements and compounds," Nuclear Instruments and Methods in Physics Research A 618, 315-322 (June 2010).

[15] Wilms, J., Allen, A., and McCray, R., "On the Absorption of X-Rays in the Interstellar Medium," ApJ 542, 914-924 (Oct. 2000).

[16] Verner, D. A., Ferland, G. J., Korista, K. T., and Yakovlev, D. G., "Atomic Data for Astrophysics. II. New Analytic FITS for Photoionization Cross Sections of Atoms and Ions," ApJ 465, 487 (July 1996). 